\title{
Effect of Malalignment on Patient Reported Outcome Measures (PROMS) following Total Knee Arthroplasty (TKA)
}

ISSN: 2576-8875

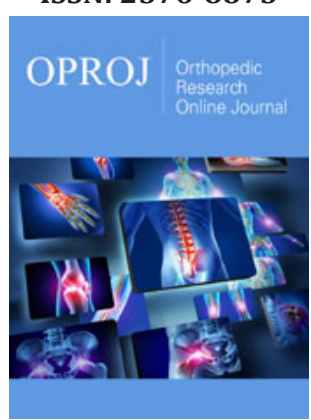

${ }^{* 1}$ Corresponding author: Mohamed Eslam Elsherif, Orthopedics Department, Assiut University Hospitals, Assiut, Egypt

Submission: 侮 March 12, 2019

Published: 眥 March 18, 2019

Volume 5 - Issue 3

How to cite this article: Mohamed $\mathrm{E} E$ Ahmed A K, Mohammad K A, Hatem B Yaser K. Effect of Malalignment on Patient Reported Outcome Measures (PROMS) following Total Knee Arthroplasty (TKA). Ortho Res Online J. 5(3). OPROJ.000611.2019.

DOI: 10.31031/OPROJ.2019.05.000611

Copyright@ Mohamed Eslam Elsherif, This article is distributed under the terms of the Creative Commons Attribution 4.0 International License, which permits unrestricted use and redistribution provided that the original author and source are credited.

\author{
Mohamed Eslam Elsherif ${ }^{*}$, Ahmed A Khalifa ${ }^{1,2}$, Mohammad Kamal Abdelnas- \\ ser $^{1}$, Hatem Bakr ${ }^{1}$ and Yaser Khalifa ${ }^{1}$ \\ ${ }^{1}$ Orthopedics Department, Assiut University Hospitals, Egypt \\ ${ }^{2}$ Orthopedics Department, South Valley University, Egypt
}

\begin{abstract}
Total knee replacement is an effective treatment for knee arthritis. While the majority of Total Knee Arthroplasties (TKAs) have proved promising long-term results, however, up to $25 \%$ of patients remain dissatisfied with the outcome of surgery at 1 year. A lack of connection between surgeons and patients in evaluating the outcome of (TKA) has led to the development of a Patient-Reported Outcome Measures (PROM) to evaluate these procedures. Implant malalignment has been involved as a contributing factor to less successful outcomes. In this review we tried to explain the evolution of the PROM concept and its different types and explaining the relationship between limb and component malalignment and PROM.
\end{abstract}

Keywords: Malalignment; Total knee arthroplasty; Outcome; PROMS

\section{Introduction}

Although primary Total Knee Arthroplasty (TKA) is one of the most frequently performed orthopaedic procedures with an excellent results and survival reaching $95 \%$ at 10 years as reported by most registries [1], its value is being subjected to increased inquiry, due both to the cost of the procedure to the healthcare system, and to frequent reports suggesting alarming rates of patient dissatisfaction with the outcome of this procedure [2-4]. So, increasing pressure is being placed on healthcare providers to demonstrate the benefits and cost-effectiveness of TKA in an objective manner [5,6]. $10 \%$ to $25 \%$ of patients are dissatisfied with the outcome of knee replacement at one to three years after surgery, mainly due to the presence of residual symptoms and the failure of the procedure to meet the patient's pre-operative expectations $[7,8]$. The outcomes of TKA have been traditionally evaluated by surgeons using invalidated scoring systems in which the patient was asked about their level of pain and return to specific activities, followed by the surgeon objectively measuring range of motion and joint stability [9]. This had led to the disturbing finding is the difference in perception of patients and their surgeons regarding the success of the procedure, with the view of surgeons often being more optimistic [10]. This has provoked a recent trend to quantify the success of this procedure using Patient-Reported Outcome Measures (PROMS) [7,8,11].

\section{What is a Patient Reported Outcome Measures (PROMs)?}

Defined by the US Food and Drug Administration as "any report of the status of a patient's health condition that comes directly from the patient [or in some cases a caregiver or surrogate], without interpretation of the patient's response by a clinician or anyone else" [12].

\section{What are the types of PROMs?}

It can be divided into either Generic or specific patient-reported outcome measures which can be distinguished by different levels of focus:

A. Generic or General health instruments are designed to provide a measure of general health for any health state, regardless of the presence or absence of illness, disability, or specific symptoms [13]. 
The most popular and well-validated general health PROMs include the Short Form 36 (SF-36) and the shorter 12-item (SF12). Both surveys include measures of physical and mental health as assessed through a number of scales. SF-36 consists of 36 items and was designed for use in clinical practice, research, health policy, and population surveys. According to the creators, it "assesses eight health concepts: (1) limitations in physical activities because of health problems; (2) limitations in social activities because of physical or emotional problems; (3) limitations in usual role activities because of health problems; (4) body pain; (5) general mental health (psychological distress and well-being); (6) limitations in usual role activities because of emotional problems; (7) vitality (energy and fatigue); and (8) general health perceptions." The survey can be self-administered by patients or administered over the phone by a trained interviewer. Administration of the 36 item questionnaires take about 5-10 minutes, but to decrease the time burden further, a shorter SF-12 was developed and validated $[14,15]$.

B. Specific PROMs focus on specific symptoms, diseases, organs, body regions, or body functions. The specific PROMs may also be specifically designed to measure the effect of a specific intervention or treatment [13].

One of the oldest and most commonly used joint-specific PROM is the Knee Society Score (KSS) [16], but it is partially surgeonderived, as the KSS combines an assessment of the knee itself (part 1) and the patient function (part 2). Part 1 includes 7 items to be assessed by the surgeon, whereas part 2 includes 3 items to be answered by the patient. The Knee Society questionnaire included items on functional ability, such as walking distance, stair climbing ability, and use of walking aids. The physical examination included assessments of the range of motion, stability, alignment, and muscle power of the knee. These data were used to calculate a Knee Society score, which consists of two scores, a knee score, and a function score, ranging from 0 to 100 points (with 100 points being the best score). The function score allocates points for walking distance and stair-climbing ability and makes deductions for the use of a walking aid; 100 represents unlimited walking distance and normal stairclimbing without the use of an aid. Fifty of the 100 points in the knee score reflect pain assessment (A score of 50 points represents no pain). The other 50 points reflect the clinical assessment of a range of motion, stability, alignment, and muscle power; 50 points represents at least $0^{\circ}$ to $125^{\circ}$ of knee flexion with no active lag, no instability, and normal alignment [17].

Wholly patient-derived instruments relevant to knee joint arthroplasty include the Western Ontario and McMaster Universities Osteoarthritis Index (WOMAC) [18] Oxford Knee Score, Knee Injury and Osteoarthritis Outcome Score (KOOS), KOOS-joint replacement (KOOS-JR) [13,15].

The Oxford Knee Score (OKS) [19,20], is a 12-item questionnaire designed to assess the impact of total knee replacement on patient pain and activity limitation. It contains questions about the level of knee pain that the patient experiences at night, during washing, drying and during use of public transport, walking time duration, the presence of limping, instability, walking down a flight of stairs. The OKS contains 12 questions, each with five categories of response. Each item is scored from 1 to 5, from least to most difficulty or severity, and combined to produce a single score with a range from 12 (least difficulties) to 60 (most difficulties) [21].

The Knee Injury and Osteoarthritis Outcome Score (KOOS) [22] was developed with the goal of creating a tool to differentiate between effective and ineffective knee treatments. The KOOS survey includes five domains of health as follows: pain, symptoms, activities of daily living, sport and recreation function, and kneerelated quality of life. The validated KOOS survey has been used in many research studies and provides a good picture of the patient's health across five dimensions. However, the survey consists of 42 items and is difficult to administer in a clinical setting because of the length of time required to complete the survey. The long length of the survey also leads to a greater possibility of missed or skipped items. So, the (KOOS-JR) was created through the identification of the items that are most relevant to the recovery of patients with TKA (page.). It consists of seven items from the pain and function domains of knee health, making it much shorter than the original KOOS [14].

Among all four specific PROMS, the KOOS uniquely accounted for ADLs related to sports and recreation. Furthermore, it was the only PROM that asked quality of life items. It is to be noted that the only alternate form of the four PROMS that was inherently distinct and not simply a cross-cultural translation of the original was the KSS which included both patient-reported, and surgeon reported, components [23].

General health and joint-specific Patient-Reported Outcome Measures (PROMs) should be used together to obtain a more complete picture of a patient's condition. The real value of PROMs, may be in helping clinicians, guide patients' treatment choices and manage expectations, thus improving the like hood of a suitable result of treatment, while physical examination and imaging are vital in making the diagnosis, PROMs assist the clinician in the frequently repeated phrase, "treat the patient, not the disease." Having a complete picture of the patients' functional limitations, pain levels, mental health state, and expectations from their treatment can help surgeons make appropriate decisions about recommending TKA to those patients most likely to benefit from the surgery. The information collected by PROMs can inform treatment decisions and facilitate communication between surgeons and patients with the potential for improving postoperative outcomes [14].

A systematic review by Ramkumar et al. [23] confirms that no single instrument exists to measure the outcome of this procedure. An optimal PROM would encompass all aspects of outcome valued by each patient and perceived as important by the healthcare provider. The instrument would broadly measure a wide range of patient demographics and expectations. However, the ideal instrument should also be specific enough to minimize the impact of comorbidities affecting the general health of the patient in distinction to the function of the knee itself. 


\section{What is an optimum alignment for TKA?}

Perfect individual component alignment in three planes should be aimed at with a neutrally aligned limb and mechanical alignment of $180^{\circ} \pm 3^{\circ}$ and no tibiofemoral rotational mismatch [24-26]. This can reduce both the mechanical stress placed on the bearing surfaces and the shear stress on the bone/prosthesis or bone/cement/prosthesis interfaces. Perfect alignment also helps to balance the forces transmitted to the soft-tissue envelope, which is crucial for proper function of the joint [27].

\section{Mechanical alignment}

The mechanical axis of the limb in the coronal plane (measured as the angle between one line drawn from the centre of the femoral head to the deepest part of the femoral notch at the knee, with a second line drawn from the midpoint of the tibial plateau to the midpoint of the inner extension of the tibiotalar joint) and its effect on function and survival had been reported more than any other alignment parameter [28,29]. The apparent benefits of achieving a neutral mechanical axis (angle of $0^{\circ}$ ) of the lower limb during TKR surgery has encouraged surgeons and manufacturers to invest time and resources into achieving this alignment target [30].

Gromov et al. [31], investigated whether the literature supports definitions of optimal alignment following primary TKA surgery and The following parameters were investigated separately: (1) mechanical tibiofemoral alignment in the AP plane; (2) tibial and femoral component placement in the AP plane; (3) tibial and femoral component placement in the sagittal plane; and (4) rotational alignment of tibial and femoral components. Although an acceptable target for alignment remains a matter for debate, most surgeons agree that the postoperative limb alignment should be corrected within $0^{\circ} \pm 3^{\circ}$ of the mechanical axis $[32,33]$.

Overall alignment has not only been linked to revision rates, but also to functional outcome as shown in a study of 115 patients by Choong et al. [34], who reported superior International Knee Society (KSS) and Short-Form 12 (SF12) scores in knees aligned within $\pm 3^{\circ}$ of neutral mechanical axis. Similar results were reported by Morgan et al. [35] in 197 patients with a mean follow-up of 9 years. They found similar revision rates in TKAs with neutral postoperative TFA (4-9 ${ }^{\circ}$ valgus) and TKAs with either varus or valgus malalignment.

Magnussen et al. [36] reported medium-term survival and KSS scores for patients with residual postoperative varus alignment (HKA $>3^{\circ}$, varus) following TKA compared to patients with neutral postoperative alignment $\left(\mathrm{HKA} 0^{\circ} \pm 3^{\circ}\right)$.

In conclusion, there have been differing reports on the importance of overall alignment on survival and functional outcome after primary TKA. As some studies used hip-knee-ankle axis (HKA) for evaluation of alignment and others used tibiofemoral axis (TFA), a direct comparison is difficult. However, neutral coronal alignment should be aimed for until there is conclusive evidence to suggest otherwise.

\section{Kinematic alignment}

Kinematic alignment was newly introduced as an alternative to conventional mechanical alignment techniques $[37,38]$. In contrast to mechanical alignment, in kinematically aligned TKA the femoral and tibial components are positioned so that the angles and the levels of the distal and posterior femoral joint line and the tibial joint line are each restored to the patient's natural alignment [37], which is not necessarily mechanically neutral [39].

The study by Dosset et al. [40], while showing improved patientreported functional outcome in TKA performed with kinematic alignment as compared to mechanically aligned TKA, only had a 6-month follow-up, making conclusions about long term outcome and survival challenging. It is entirely possible that a kinematically aligned, but mechanically mal-aligned implanted prosthesis could recreate a patient's preoperative kinematics [41]. Howell et al. [42] concluded that kinematic aligned knee replacement did not adversely affect implant survival or function as it restores the constitutional alignment of the limb and joint line, subsequently avoiding collateral ligament imbalances [42]. This would create a group of patients that maybe considered mechanically "malaligned", but had good PROMs scores based on their individual alignment [41].

More studies are therefore needed to study whether kinematic alignment can produce better survivorship and functional outcome following primary TKA.

\section{Does malalignment affect PROMS?}

It is suggested that the evidence of poor outcomes secondary to malalignment is largely historic based on studies of inferior implant designs, and the use of poor radiological techniques when assessing malalignment, also the choice of target for ideal alignment has been challenged by proponents of kinematically aligned TKA who have reported promising results $[33,37,43,44]$. Implant malalignment following primary TKA has been reported to be the primary reason for revision in $7 \%$ of revised TKAs [45], and it has been linked to both decreased implant survival [46], and inferior patient-reported outcomes [34,47]. Surgeon-dependent factors such as ideal implant alignment are thought to play an important role in outcome following primary total knee arthroplasty (TKA). Malalignment can occur either in the coronal, sagittal or axial plane and even can be a combination of the three.

\section{Coronal malalignment}

Coronal limb malalignment is considered as one of the most important factors determining long-term prosthesis survival. Several authors stressed the importance of restoring limb coronal mechanical alignment to within $180^{\circ}$ [30]. Sagittal malalignment may result in an overstuffing and limited joint range of motion. Axial component malalignment can result in abnormal patellar tracking and subsequent anterior knee pain [41]. Although Several authors stressed the importance of restoring limb coronal mechanical alignment to within $180^{\circ}$, in a systematic review showed that about 
$64 \%$ of studies investigating alignment in the coronal plane showed no association between malalignment and worse patient-reported outcome measures [41].

\section{Sagittal malalignment}

Components malalignment on this plane can alter the posterior tibial slop and affect the flexion and extension gaps. This may result in overstuffing and limited joint range. Femoral notching can be seen in the excessive femoral component extension position. Lustig et al. [48] studied 95 patients, showed that sagittal placement of the femoral component predicts flexion contracture, as the posterior slope of $>3.5^{\circ}$ from the mechanical axis increased the risk of mild flexion contracture at 1 -year follow-up by 3 timesindependently of other variables. Kim et al. [49] showed sagittal malpositioning of the femoral component has also been linked to inferior survival, as a $3.3 \%$ failure rate in knees with femoral implant flexed $>3^{\circ}$ as compared to failure rates of $0 \%$ and $0.9 \%$ in neutrally aligned femoral components $\left(0-3^{\circ}\right.$ flexion $)$ and extended femoral components ( $>1^{\circ}$ extension), respectively. The same study also found that tibial malalignment in the sagittal plane $\left(<0^{\circ}\right.$ or $\left.>7^{\circ}\right)$ had a failure rate of $4.5 \%$, as compared to a failure rate of $0.2 \%$ in the neutrally-aligned group.

In conclusion, few studies have investigated the role of sagittal component positioning in TKA surgery. It appears that to improve the survival rate and functional outcome following TKA, the surgeon should aim to place the femoral component in $0-3^{\circ}$ of flexion and posterior tibial slope should be $0-7^{\circ}$ [31]. However, $100 \%$ of studies reviewed in this review showed no associated between sagittal malalignment and worse outcome measures [41].

\section{Axial malalignment}

Many references exist for measuring femoral and tibia component rotation. Individual component malalignment and the combined mismatch can result in abnormal patella tracking and subsequent anterior knee pain.

Cadaver studies have shown that optimal patella tracking is achieved when the femoral component is in a neutral position or externally rotated [50]. These findings were confirmed by Matsuda et al. [51] who showed a correlation between the internal rotation of the femoral component, patellar tilt angle and clinical symptoms. Also, Kelly et al. [52] had found that placing the femoral component in internal rotation, will displace the patella medially, thus increasing the $Q$ angle, which can, in turn, lead to lateral patellar tilt, lateral patellar overhang, subluxation, and dislocation. Also, Kim et al. [49], had reported an increased failure rate in femoral components with $<2^{\circ}$ of external rotation (6.7\% failure) and also in femoral components with $>5^{\circ}$ of external rotation (1.9\% failure) when compared to components with $2-5^{\circ}$ of external rotation $(0 \%$ failure) in relation to STEA, suggesting that $2-5^{\circ}$ of external rotation is the optimal position. However, a study by Bell et al. [53] on 56 TKA patients with unexplained knee pain showed that an internally rotated femoral component $\left(>0.3^{\circ}\right.$ internally rotated in relation to the surgical transepicondyler axis, sTEA) was a significant factor in pain following TKA. Murakami et al. [54] also reported a correlation between pain following TKA and internal rotation of the femoral component $\left(>0^{\circ}\right.$ internally rotated in relation to sTEA). Nicoll et al. [55] compared painful and pain-free TKAs and found that internal rotation of the tibial component by $>9^{\circ}$ (in relation to neutral tibial tuberosity axis (TTA) was a major cause of pain and functional deficit following TKA. The same study did not find external rotational errors to be associated with pain. Barrack et al. [56] investigated 102 TKAs with a minimum follow-up of 5 years and found significant differences in tibial component rotation between the 2 groups, with patients with anterior knee pain averaging $6^{\circ}$ of internal rotation (in relation to neutral TTA) as compared to $0.4^{\circ}$ of external rotation in the control group. Bell et al. [53] found that an internally rotated tibial component (internally in relation to neutral TTA) was a substantial factor for pain following TKA. Kim et al. [49] showed that rotational alignment of the tibial component by $<2^{\circ}$ or $>5^{\circ}$ of external rotation (in relation to posterior margins of the tibial plateau) increased component failure rates. Excessive internal rotation, when measured in relation to the tibial tuberosity, can lead to knee pain [31]. Hadi et al. [41] review shows $50 \%$ of studies found an association between malalignment and worse PROMs [41].

\section{Did computer-assisted TKA offer better PROMS?}

Although Computer-navigated TKR is reported to improve the overall accuracy of positioning of the tibial and femoral components with better overall alignment $[44,57,58]$. But have demonstrated little evidence of a clinical advantage [44,59].

As Spencer and colleagues [60] found no statistically significant difference in patient satisfaction comparing computer-navigated TKA and conventional TKA 2 years after surgery. Similarly, Harvie and colleagues reported no difference in patient satisfaction comparing computer-navigated TKA versus conventional TKA at a period of 5 years after surgery. It would appear that computer navigation does not have an effect on patient satisfaction [61].

There have been many trials that have evaluated radiological alignment following total knee arthroplasty which has shown improved outcomes with CAS [62,63]. Fewer studies, however, have looked at functional outcomes: a randomized trial of 52 patients by Decking et al. [64] compared CAS with conventional surgery. They failed to detect a significant difference in the Knee Society Score (KSS), the WOMAC score and range of motion at 3-month follow-up [64]. Similarly, Matziolis et al. [44] found no difference in the range of motion and KSS at 6 months in a randomized trial of 60 patients. A trial by Kim et al. looked at sequential simultaneous bilateral total knee arthroplasties in 160 patients (320 knees). One knee replacement was performed using CAS and the other was performed using conventional instruments. There was no significant difference seen in alignment. Hospital for Special Surgery (HSS) knee scores, KSS, and range of motion of the knee demonstrated no difference between the groups at three months, one year, and a mean of 3.4 years after the operation [65].

\section{Conclusion}

Patient-reported outcome measures reflected the current change of focus from volume-based to value-based healthcare delivery. Measuring patient-reported outcomes have provided 
important information about what matters to patients, the early Patient-Reported Outcome Measures (PROMs) have been shown to be affected mainly by component malalignment in the axial plane but not significantly affected by Limb malalignment in both coronal and sagittal planes. Component position can't be simplified by describing it as "mal-aligned" or "aligned" as a complex relationship between all measures of limb and component alignment (in both the tibial and the femoral components) play a role in the final procedure results and subsequent implant survival and patients reported outcomes.

\section{References}

1. Graves SE, Davidson D, Ingerson L, Ryan P, Griffith EC, et al. (2004) The Australian orthopaedic association national joint replacement registry. Medical Journal of Australia180(5): S31-34.

2. Noble PC, Conditt MA, Cook KF, Mathis KB (2006) The john insall award: Patient expectations affect satisfaction with total knee arthroplasty. Clinical Orthopaedics and Related Research (1976-2007). 452: 35-43.

3. Alviar MJ, Olver J, Brand C, Hale T, Khan F (2011) Do patient-reported outcome measures used in assessing outcomes in rehabilitation after hip and knee arthroplasty capture issues relevant to patients? Results of a systematic review and ICF linking process. Journal of rehabilitation medicine 43(5): 374-381.

4. Collins NJ, Roos EM (2012) Patient-reported outcomes for total hip and knee arthroplasty: commonly used instruments and attributes of a "good" measure. Clinics in geriatric medicine 28(3): 367-394.

5. Jones CA, Beaupre LA, Johnston D, Suarez-Almazor ME (2007) Total joint arthroplasties: current concepts of patient outcomes after surgery. Rheumatic Disease Clinics of North America 33(1): 71-86.

6. Jourdan C, Poiraudeau S, Descamps S, Nizard R, Hamadouche M, et al. (2012) Comparison of patient and surgeon expectations of total hip arthroplasty. PloS one 7(1): e30195.

7. Bourne RB (2008) Measuring tools for functional outcomes in total knee arthroplasty. Clinical orthopaedics and related research 466(11): 26342638.

8. Ghomrawi HM, Ferrando NF, Mandl LA, Do H, Noor N, et al. (2011) How often are patient and surgeon recovery expectations for total joint arthroplasty aligned? Results of a pilot study. HSS journal 7(3): 229-234.

9. Bourne RB, Chesworth BM, Davis AM, Mahomed NN, Charron KD (2010) Patient satisfaction after total knee arthroplasty: who is satisfied and who is not? Clinical Orthopaedics and Related Research 468(1): 57-63.

10. Howick J (2011) The oxford 2011 levels of evidence

11. Seil R, Pape D (2011) Causes of failure and etiology of painful primary total knee arthroplasty. Knee Surgery, Sports Traumatology, Arthroscopy 19(9): 1418-1432.

12. Health UDo, Services H (2009) Guidance for industry-Patient-reported outcome measures: Use in medical product development to support labeling claims.

13. Rolfson O, Bohm E, Franklin P, Lyman S, Denissen G, et al. (2016) Patient-reported outcome measures in arthroplasty registries: Report of the patient-reported outcome measures working group of the international society of arthroplasty registries part II. Recommendations for selection, administration, and analysis. Acta orthopaedical 87(sup1): 9-23.

14. Lyman S, Yin KL (2017) Patient-reported outcome measurement for patients with total knee arthroplasty. The Journal of the American Academy of Orthopaedic Surgeons 25 Suppl 1: S44-s47.

15. Lyman S, Hidaka C (2016) Patient-reported outcome measures-what data do we really need? J Arthroplasty 31(6): 1144-1147.
16. Perruccio AV, Lohmander LS, Canizares M, Tennant A, Hawker GA, et al. (2008) The development of a short measure of physical function for knee OA KOOS-Physical Function Shortform (KOOS-PS)-an OARSI/ OMERACT initiative. Osteoarthritis and cartilage 16(5): 542-550.

17. Lingard EA, Katz JN, Wright RJ, Wright EA, Sledge CB, et al. (2001) Validity and responsiveness of the knee society clinical rating system in comparison with the SF-36 and WOMAC. Journal of Bone and Joint Surgery (American Volume) 83(12): 1856-1864.

18. Bellamy N, Buchanan WW, Goldsmith CH, Campbell J, Stitt LW (1988) Validation study of WOMAC: a health status instrument for measuring clinically important patient relevant outcomes to antirheumatic drug therapy in patients with osteoarthritis of the hip or knee. The Journal of rheumatology 15(12): 1833-1840.

19. Dawson J, Fitzpatrick R, Murray D, Carr A (1998) Questionnaire on the perceptions of patients about total knee replacement. The Journal of bone and joint surgery British volume 80(1): 63-69.

20. Murray D, Fitzpatrick R, Rogers K, Pandit H, Beard D, et al. (2007) The use of the Oxford hip and knee scores. The Journal of bone and joint surgery British volume 89(8): 1010-1014.

21. Dawson J, Fitzpatrick R, Murray D, Carr A (1998) Questionnaire on the perceptions of patients about total knee replacement. The Journal of bone and joint surgery British volume 80(1): 63-69.

22. Roos EM, Roos HP, Lohmander LS, Ekdahl C, Beynnon BD (1998) Knee Injury and Osteoarthritis Outcome Score (KOOS)-development of a self-administered outcome measure. Journal of Orthopaedic \& Sports Physical Therapy 28(2): 88-96.

23. Ramkumar P, Harris J, Noble P (2015) Patient-reported outcome measures after total knee arthroplasty: a systematic review. Bone \& joint research 4(7): 120-127.

24. Sikorski J (2008) Alignment in total knee replacement. The Journal of bone and joint surgery British volume 90(9): 1121-1127.

25. Ritter MA, Faris PM, Keating EM, Meding JB (1994) Postoperative alignment of total knee replacement. Its effect on survival. Clinical orthopaedics and related research (299): 153-156.

26. Longstaff LM, Sloan K, Stamp N, Scaddan M, Beaver R (2009) Good alignment after total knee arthroplasty leads to faster rehabilitation and better function. The Journal of arthroplasty 24(4): 570-578.

27. Pickering S, Armstrong D (2012) Focus on alignment in total knee replacement. Journal of Bone and Joint Surgery (British Volume) 2012: $1-3$.

28. Jeffery RS, Morris RW, Denham RA (1991) Coronal alignment after total knee replacement. The Journal of bone and joint surgery British volume 73(5): 709-714.

29. Vince KG, Insall JN, Kelly MA (1989) The total condylar prosthesis. 10-to 12 -year results of a cemented knee replacement. The Journal of bone and joint surgery British volume 71(5): 793-797.

30. Oswald MH, Jakob RP, Schneider E, Hoogewoud HM (1993) Radiological analysis of normal axial alignment of femur and tibia in view of total knee arthroplasty. The Journal of arthroplasty 8(4): 419-426.

31. Gromov K, Korchi M, Thomsen MG, Husted H, Troelsen A (2014) What is the optimal alignment of the tibial and femoral components in knee arthroplasty? An overview of the literature. Acta orthopaedical 85(5): 480-487.

32. Parratte S, Pagnano MW, Trousdale RT, Berry DJ (2010) Effect of postoperative mechanical axis alignment on the fifteen-year survival of modern, cemented total knee replacements. Journal of Bone and Joint Surgery (American Volume) 92(12): 2143-2149.

33. Bonner T, Eardley W, Patterson P, Gregg P (2011) The effect of post-operative mechanical axis alignment on the survival of primary total knee replacements after a follow-up of 15 years. J Bone Joint Surg Br 93(9): $1217-1222$ 
34. Choong PF, Dowsey MM, Stoney JD (2009) Does accurate anatomical alignment result in better function and quality of life? Comparing conventional and computer-assisted total knee arthroplasty. J Arthroplasty 24(4): 560-569.

35. Morgan SS, Bonshahi A, Pradhan N, Gregory A, Gambhir A, et al. (2008) The influence of postoperative coronal alignment on revision surgery in total knee arthroplasty. International orthopaedics 32(5): 639-642.

36. Magnussen RA, Weppe F, Demey G, Servien E, Lustig S (2011) Residual varus alignment does not compromise results of TKAs in patients with preoperative varus. Clinical Orthopaedics and Related Research 469(12): 3443-3450.

37. Howell SM, Howell SJ, Kuznik KT, Cohen J, Hull ML (2013) Does a kinematically aligned total knee arthroplasty restore function without failure regardless of alignment category? Clinical Orthopaedics and Related Research 471(3): 1000-1007.

38. Howell SM, Papadopoulos S, Kuznik KT, Hull ML (2013) Accurate alignment and high function after kinematically aligned TKA performed with generic instruments. Knee Surgery, Sports Traumatology, Arthroscopy 21(10): 2271-2280.

39. Eckhoff DG, Bach JM, Spitzer VM, Reinig KD, Bagur MM, et al. (2005) Three-dimensional mechanics, kinematics, and morphology of the knee viewed in virtual reality. Journal of Bone and Joint Surgery (American Volume) 87: 71-80.

40. Dossett HG, Swartz GJ, Estrada NA, LeFevre GW, Kwasman BG (2012) Kinematically versus mechanically aligned total knee arthroplasty. Orthopedics 35(2): e160-e169.

41. Hadi M, Barlow T, Ahmed I, Dunbar M, McCulloch P, et al. (2016) Does malalignment affect patient reported outcomes following total knee arthroplasty: a systematic review of the literature. Springerplus 5(1):1201.

42. Howell SM, Papadopoulos S, Kuznik K, Ghaly LR, Hull ML (2015) Does varus alignment adversely affect implant survival and function six years after kinematically aligned total knee arthroplasty? International orthopaedics 39(11): 2117-2124.

43. Bach CM, Mayr E, Liebensteiner M, Gstottner M, Nogler M, et al. (2009) Correlation between radiographic assessment and quality of life after total knee arthroplasty. The Knee 16(3): 207-210.

44. Matziolis G, Adam J, Perka C (2010) Varus malalignment has no influence on clinical outcome in midterm follow-up after total knee replacement. Archives of orthopaedic and trauma surgery 130(12): 1487-1491.

45. Schroer WC, Berend KR, Lombardi AV, Barnes CL, Bolognesi MP, et al. (2013) Why are total knees failing today? Etiology of total knee revision in 2010 and 2011. J Arthroplasty 28(8): 116-119.

46. Ritter MA, Davis KE, Meding JB, Pierson JL, Berend ME, et al. (2011) The effect of alignment and BMI on failure of total knee replacement. Journal of Bone and Joint Surgery (American Volume) 93(17): 1588-1596.

47. Longstaff LM, Sloan K, Stamp N, Scaddan M, Beaver R (2009) Good alignment after total knee arthroplasty leads to faster rehabilitation and better function. The Journal of arthroplasty 24(4): 570-578.

48. Lustig S, Scholes CJ, Stegeman TJ, Oussedik S, Coolican MR, et al. (2012) Sagittal placement of the femoral component in total knee arthroplasty predicts knee flexion contracture at one-year follow-up. International orthopaedics 36(9): 1835-1839.

49. Kim YH, Park JW, Kim JS, Park SD (2014) The relationship between the survival of total knee arthroplasty and postoperative coronal, sagittal and rotational alignment of knee prosthesis. International orthopaedics 38(2): 379-385.
50. Anouchi YS, Whiteside LA, Kaiser AD, Milliano MT (1993) The effects of axial rotational alignment of the femoral component on knee stability and patellar tracking in total knee arthroplasty demonstrated on autopsy specimens. Clinical orthopaedics and related research 1993(287): 170-177.

51. Matsuda S, Miura H, Nagamine R, Urabe K, Hirata G, et al. (2001) Effect of femoral and tibial component position on patellar tracking following total knee arthroplasty: 10-year follow-up of Miller-Galante I knees. American journal of knee surgery 14(3): 152-156.

52. Kelly M (2001) Patellofemoral complications following total knee arthroplasty. Instructional course lectures 50: 403-407.

53. Bell SW, Young P, Drury C, Smith J, Anthony I, et al. (2014) Component rotational alignment in unexplained painful primary total knee arthroplasty. The Knee 21(1): 272-277.

54. Murakami A, Hash T, Hepinstall M, Lyman S, Nestor B, et al. (2012) MRI evaluation of rotational alignment and synovitis in patients with pain after total knee replacement. J Bone Joint Surg Br 94(9): 1209-1215.

55. Nicoll D, Rowley DI (2010) Internal rotational error of the tibial component is a major cause of pain after total knee replacement. J Bone Joint Surg Br 92(9): 1238-1244.

56. Barrack RL, Schrader T, Bertot AJ, Wolfe MW, Myers L (2001) Component rotation and anterior knee pain after total knee arthroplasty. Clinical orthopaedics and related research 392: 46-55.

57. Stöckl B, Nogler M, Rosiek R, Fischer M, Krismer M, et al. (2004) Navigation improves accuracy of rotational alignment in total knee arthroplasty. Clinical orthopaedics and related research 426: 180-186.

58. Bäthis H, Perlick L, Tingart M, Lüring C, Zurakowski D, et al. (2004) Alignment in total knee arthroplasty: a comparison of computer-assisted surgery with the conventional technique. The Journal of bone and joint surgery British volume 86(5): 682-687.

59. Cheng T, Pan XY, Mao X, Zhang GY, Zhang XL (2012) Little clinical advantage of computer-assisted navigation over conventional instrumentation in primary total knee arthroplasty at early follow-up. The Knee 19(4): 237-245.

60. Spencer J, Chauhan S, Sloan K, Taylor A, Beaver R (2007) Computer navigation versus conventional total knee replacement: no difference in functional results at two years. The Journal of bone and joint surgery British volume 89(4): 477-480.

61. Harvie P, Sloan K, Beaver RJ (2012) Computer navigation vs conventional total knee arthroplasty: five-year functional results of a prospective randomized trial. The Journal of arthroplasty 27(5): 667-672.

62. Cheng T, Zhao S, Peng X, Zhang X (2012) Does computer-assisted surgery improve postoperative leg alignment and implant positioning following total knee arthroplasty? A meta-analysis of randomized controlled trials? Knee Surgery, Sports Traumatology, Arthroscopy 20(7): 1307-1322.

63. Bauwens K, Matthes G, Wich M, Gebhard F, Hanson B, et al. (2007) Navigated total knee replacement: a meta-analysis. JBJS 89(2): 261-269.

64. Decking R, Markmann Y, Fuchs J, Puhl W, Scharf HP (2005) Leg axis after computer-navigated total knee arthroplasty: a prospective randomized trial comparing computer-navigated and manual implantation. The Journal of Arthroplasty 20(3): 282-288.

65. Kim YH, Kim JS, Choi Y, Kwon OR (2009) Computer-assisted surgical navigation does not improve the alignment and orientation of the components in total knee arthroplasty. JBJS 91(1): 14-19. 